Delft University of Technology

\title{
Thermal Properties of NbN Single-Photon Detectors
}

Baeva, E. M.; Sidorova, M. V.; Korneev, A. A.; Smirnov, K. V.; Divochy, A. V.; Morozov, P. V.; Zolotov, P. I.; Vakhtomin, Yu B.; Klapwijk, T. M.; More Authors

DOI

10.1103/PhysRevApplied.10.064063

Publication date

2018

Document Version

Final published version

Published in

Physical Review Applied

\section{Citation (APA)}

Baeva, E. M., Sidorova, M. V., Korneev, A. A., Smirnov, K. V., Divochy, A. V., Morozov, P. V., Zolotov, P. I., Vakhtomin, Y. B., Klapwijk, T. M., \& More Authors (2018). Thermal Properties of NbN Single-Photon

Detectors. Physical Review Applied, 10(6), [064063]. https://doi.org/10.1103/PhysRevApplied.10.064063

\section{Important note}

To cite this publication, please use the final published version (if applicable).

Please check the document version above.

\section{Copyright}

Other than for strictly personal use, it is not permitted to download, forward or distribute the text or part of it, without the consent of the author(s) and/or copyright holder(s), unless the work is under an open content license such as Creative Commons.

Takedown policy

Please contact us and provide details if you believe this document breaches copyrights.

We will remove access to the work immediately and investigate your claim. 


\title{
Thermal Properties of NbN Single-Photon Detectors
}

\author{
E.M. Baeva, ${ }^{1,2}$ M.V. Sidorova, ${ }^{2,3}$ A.A. Korneev, ${ }^{1,2}$ K.V. Smirnov, ${ }^{1,2,4}$ A.V. Divochy, ${ }^{2,4}$ P.V. Morozov, ${ }^{4}$ \\ P.I. Zolotov, ${ }^{1,2,4}$ Yu.B. Vakhtomin, ${ }^{2,4}$ A.V. Semenov, ${ }^{2}$ T.M. Klapwijk, ${ }^{2,5}$ V.S. Khrapai, ${ }^{1,2,{ }^{*}}$ and \\ G.N. Goltsman ${ }^{1,2}$ \\ ${ }^{1}$ National Research University Higher School of Economics, 20 Myasnitskaya Street, Moscow 101000, Russia \\ ${ }^{2}$ Moscow State University of Education, 29 Malaya Pirogovskaya Street, Moscow 119435, Russia \\ ${ }^{3}$ DLR Institute of Optical Systems, Rutherfordstrasse 2, 12489 Berlin, Germany \\ ${ }^{4}$ LLC Superconducting nanotechnology (Scontel), 5/22 Rossolimo Street, Moscow 119021, Russia \\ ${ }^{5}$ Kavli Institute of Nanoscience, Delft University of Technology, 2628 CJ Delft, Netherlands
}

(Received 7 October 2018; revised manuscript received 4 December 2018; published 28 December 2018)

\begin{abstract}
We investigate thermal properties of a $\mathrm{NbN}$ single-photon detector capable of unit internal detection efficiency. Using an independent calibration of the coupling losses, we determine the absolute optical power absorbed by the $\mathrm{NbN}$ film and, via resistive superconductor thermometry, the temperature dependence of the thermal resistance $Z(T)$ of the $\mathrm{NbN}$ film. In principle, this approach permits simultaneous measurement of the electron-phonon and phonon-escape contributions to the energy relaxation, which in our case is ambiguous because of the similar temperature dependencies. We analyze $Z(T)$ with a two-temperature model and impose an upper bound on the ratio of electron and phonon heat capacities in $\mathrm{NbN}$, which is surprisingly close to a recent theoretical lower bound for the same quantity in similar devices.
\end{abstract}

DOI: 10.1103/PhysRevApplied.10.064063

\section{INTRODUCTION}

State-of-the-art superconducting single-photon detectors (SSPDs) are sensitive to infrared photons, have near$100 \%$ detection efficiency [1-3], and have a counting rate of hundreds of megahertz [4], making them suitable for a wide range of applications [5]. To combine all these high performances in one device, however, is a challenge and requires not only technical advancements but also a clear understanding of the physics behind single-photon detection; for a recent review, see Ref. [6].

The problem of SSPD theory lies in a complex interplay of resistive and thermal properties of a thin superconducting film under strong nonequilibrium [7]. Understanding the resistive properties has two major complications. First, the superconducting films usually used in SSPDs are strongly disordered, with a sheet resistance in the range approaching a superconductor-insulator transition [8]. Second, the physics of the resistive transition in thin superconducting films is closely related to the BerezinskiiKosterlitz-Thouless (BKT) theory [9,10], which connects the temperature dependence of the resistance with the density of free topological excitations - superconducting vortices. The thermal aspect is yet-more complex, for the conversion of the absorbed photon energy in a

\footnotetext{
*dick@issp.ac.ru
}

current-biased film interconnects the electronic excitations, the Cooper-pair condensate with vortices, and the phonon system.

The very beginning of the detection event manifests itself in a highly nonequilibrium region within the superconducting film, usually called the "hot spot" (HS). In the simplest interpretation of a so-called geometric HS model [11], the role of the HS is to create a normal conducting region, which causes a redistribution of the current density incompatible with the zero-resistance state. Recent theoretical work $[12,13]$ considers the situation as much less trivial owing to a role played by the current-crowding effect and the superconducting vortices, accordingly dubbed in Ref. [14] the "photon-generated-superconducting-vortex model." In this model, the emerging HS captures a single vortex or a vortex-antivortex pair, depending on the position of the HS across the superconducting strip. Above a certain threshold bias current, the vortices can unbind from the HS and pass across the strip in an avalanche manner, causing the dissipation and expansion of the HS. The detection efficiency of a SSPD depends on how the initial photon energy is redistributed between the electron and phonon systems throughout the HS evolution. The photongenerated-superconducting-vortex model [13] relates the efficiency to the ratio of the heat capacities of electron and phonon systems in a universal way, which emphasizes the role of thermal properties of a superconductor used in a SSPD. 
In this work, we analyze a NbN-nanowire-based SSPD capable of unit internal detection efficiency in terms of its thermal response under continuous irradiation with infrared light. The key ingredient of our approach is knowledge of the absolute amount of energy absorbed by the film, which is achieved via an independent calibration of the apparatus's coupling losses. Using resistive superconductor thermometry in zero and finite magnetic fields, we obtain the thermal resistance $Z(T)$ of a $\mathrm{NbN}$ film and analyze it with a two-temperature model. Potentially, our approach allows us to extract the individual contributions of the electron-phonon relaxation and the phonon escape into the substrate to $Z(T)$. This is difficult in the present experiment because of the similar temperature dependencies of the two contributions. Instead, we impose an experimental upper bound on the ratio of electron and phonon heat capacities, which is surprisingly consistent with the lower bound implied by the model calculations for similar devices [13].

\section{METHODS AND EXPERIMENTAL DETAILS}

\section{A. Two-temperature model}

As discussed above, the resistive response of a SSPD is based on a complex nonequilibrium process of conversion of the absorbed photon energy into the energy of the Cooper-pair condensate with superconducting vortices, the electronic excitations, and the phonon system. Focusing here on the thermal properties in the vicinity of the resistive transition, we use a more reduced approach and consider the system in terms of the commonly used twotemperature model (Fig.1). With certain reservations (see Sec. II D for details), one can ignore the spatially inhomogeneous nature of the photon absorption and assume uniform, yet different, temperatures for the electrons, $T_{e}$, and the (acoustic) phonons, $T_{\mathrm{ph}}$. The radiation energy is absorbed by the electrons and is transferred to the substrate in a time called the "energy relaxation time," which can be measured via amplitude-modulated absorption of terahertz radiation (AMAR) [15]. This method was introduced by Gershenzon et al. [16] and has since then been applied to various superconducting materials with different temperature dependencies of the relaxation time; for instance, $T^{-2}$ in $\mathrm{Nb}[16]$ and boron-doped diamond [15], $T^{-1.6}$ in $\mathrm{NbN}$ $[17,18]$, and $T^{-3}$ in TiN [19], NbC [20], and WSi [21] films.

Similarly to the AMAR approach, here we also maintain the sample at a bath temperature $T_{b}$ close to the resistive transition and bias it with a direct current $I$ to measure the change of the resistance in response to the applied radiation, which is referred to as "resistive superconductor thermometry" above. We assume that the current is small enough, such that there is a one-to-one thermodynamic correspondence between an increase in $T_{e}$ and the film resistance. In the BKT scenario, the resistance is (a)

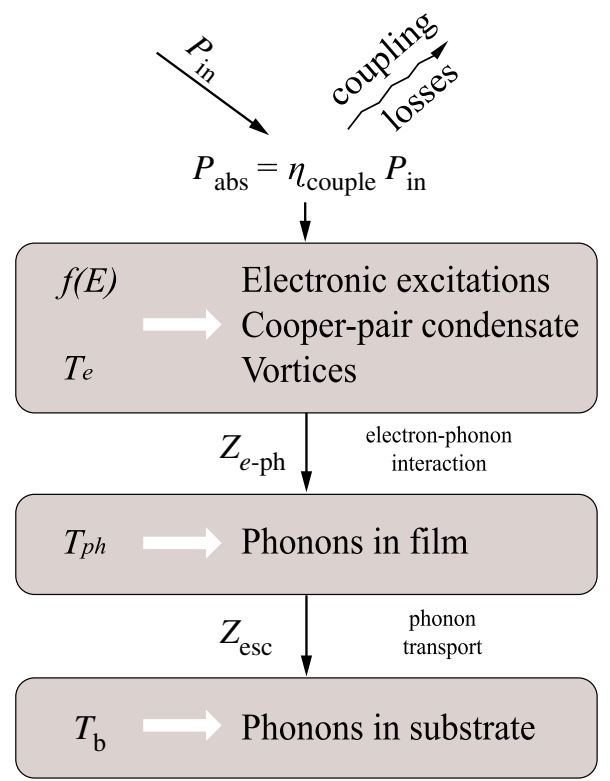

(b)

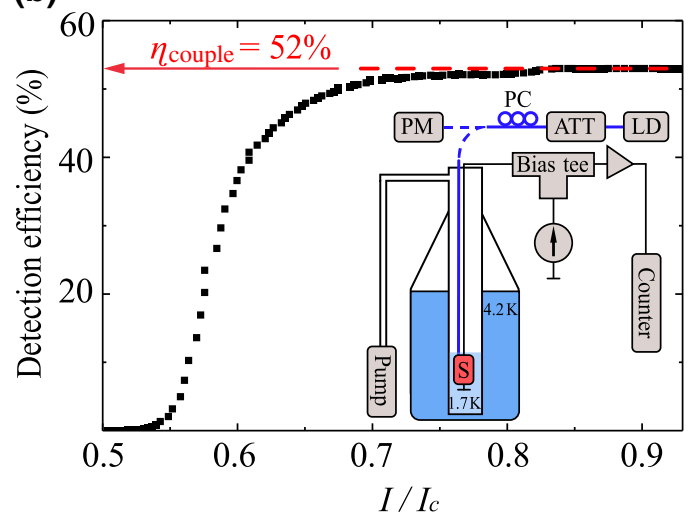

FIG. 1. (a) Two-temperature model of a metal-superconducting film on an insulating substrate. Only a fraction of the incident optical power $P_{\text {in }}$ is absorbed by the film as determined by the coupling efficiency of the apparatus $P_{\text {abs }}=\eta_{\text {couple }} P_{\text {in }}$. The electron subsystem under illumination can be described by an equilibrium Fermi-Dirac distribution with an effective electron temperature $T_{e}$ exceeding the phonon temperature in $\mathrm{NbN} T_{\mathrm{ph}}$ and the bath temperature $T_{b}$. Further, it is assumed that $T_{e}$ is the only parameter that controls the resistivity of the superconducting film. $Z_{e \text {-ph }}$ and $Z_{\text {esc }}$ are the thermal resistances, which determine the heat exchange of the electrons with the acoustic phonons in $\mathrm{NbN}$ and the heat exchange of the phonons in $\mathrm{NbN}$ with the substrate, respectively. (b) Measured SSPD detection efficiency $D_{E}$ at a wavelength of $1.55 \mu \mathrm{m}$ at $T_{b}=1.7 \mathrm{~K}$ versus the bias current in the unit of the critical current. The saturation of the bias-current dependence signals the regime of unit internal efficiency, which enables us to calibrate the coupling losses as $D_{E}=\eta_{\text {couple }}$. The inset shows a sketch of the experimental setup. ATT, attenuator; LD, laser diode; PC, polarization controller; PM, power meter; S, sample.

determined by the number of free superconducting vortices and antivortices, which are equally populated in zero magnetic field and preferably polarized in one direction in a finite magnetic field [22]. We therefore assume that the 
change in the vortex density caused by both the direct current and the absorbed optical power is negligible compared with its equilibrium value, which is justified in the vicinity of the resistive transition, where the topological excitations are macroscopically populated. An important difference from the AMAR case is that in present experiment the modulation frequency of the radiation is much smaller than the intrinsic relaxation rate in NbN SSPDs, since we are interested in a time-averaged and device-averaged resistive response, which is a measure of the thermal resistance between the electron system in $\mathrm{NbN}$ and the bulk substrate.

In the experiment we use the resistive properties of the superconductor as a function of $T_{e}$. In the vicinity of the resistive transition, where phase coherence breaks down, we assume that the resistivity of the superconductor is a direct measure of $T_{e}$. In the two-temperature model, the time-dependent temperatures of the electrons $T_{e}$ and phonons $T_{\mathrm{ph}}$ are obtained from the following coupled linear heat-balance equations [23,24]:

$$
\begin{gathered}
C_{e} \frac{d T_{e}}{d t}=P_{\mathrm{abs}}+P_{\mathrm{dc}}-\frac{C_{e}}{\tau_{e-\mathrm{ph}}}\left(T_{e}-T_{\mathrm{ph}}\right), \\
C_{\mathrm{ph}} \frac{d T_{\mathrm{ph}}}{d t}=\frac{C_{\mathrm{ph}}}{\tau_{\mathrm{ph}-e}}\left(T_{e}-T_{\mathrm{ph}}\right)-\frac{C_{\mathrm{ph}}}{\tau_{\mathrm{esc}}}\left(T_{\mathrm{ph}}-T_{b}\right),
\end{gathered}
$$

where $T_{b}$ is the bath temperature, $P_{\mathrm{dc}}=I U$ is the Joule heat power and $P_{\text {abs }}$ is the power of the applied radiation absorbed by the film, $\tau_{e \text {-ph }}$ is the electron-phonon time, $\tau_{\text {ph-e }}$ is the phonon-electron time, and $\tau_{\text {esc }}$ is the phonon escape time. Note that the usual equilibrium relation $C_{\mathrm{ph}} / \tau_{\mathrm{ph}-e}=$ $C_{e} / \tau_{e-\text { ph }}$ also holds here.

The differential equations (1) and (2) are further simplified under the conditions of our experiment. The first is the steady-state condition $d T_{i} / d t=0$, since the modulation period of the incident radiation, $1 / f=1 / 26 \mathrm{~Hz}=38$ $\mathrm{ms}$, is much greater than the typical time constants in dirty $\mathrm{NbN}$; that is, the energy relaxation time $(10-20 \mathrm{ps})$ $[18,25]$ and the phonon escape time (approximately 50 ps) [26]. The second is the condition of small bias current, which allows us to ignore Joule heating, $P_{\mathrm{dc}} \ll P_{\mathrm{abs}}$, and minimize the potential effect of the current on the creation of extra free vortices beyond the equilibrium number determined by the electron temperature (not included in the model above). As such, the model reduces to a single equation for the total thermal resistance, $Z \equiv\left(T_{e}-\right.$ $\left.T_{b}\right) / P_{\text {abs }}$ :

$$
Z=\frac{\tau_{e-\mathrm{ph}}}{C_{e}}+\frac{\tau_{\mathrm{esc}}}{C_{\mathrm{ph}}}=Z_{e-\mathrm{ph}}+Z_{\mathrm{esc}} .
$$

Equation (3) intuitively expresses the two-stage transfer of the heat from the electronic system toward the equilibrium bath in our two-temperature model, as schematically presented in Fig. 1(a). The absorbed radiation power raises the electron temperature $T_{e}$ with respect to the acoustic phonon temperature $T_{\mathrm{ph}}$ in the $\mathrm{NbN}$ film. The difference between $T_{e}$ and $T_{\mathrm{ph}}$ is determined by the first contribution in Eq. (3), which is the electron-phonon thermal resistance in NbN, $Z_{e \text {-ph }} \equiv \tau_{e \text {-ph }} / C_{e}$. In turn, the finite escape time for escape of the acoustic phonons from $\mathrm{NbN}$ into the substrate causes an increase of $T_{\mathrm{ph}}$ compared with the bath temperature $T_{b}$, which results in the second contribution to the thermal resistance, $Z_{\mathrm{esc}} \equiv \tau_{\mathrm{esc}} / C_{\mathrm{ph}}$.

In what follows we are interested in the ratio of the normal-state thermal capacity of the electronic system, $C_{e}^{n}$, and the thermal capacity of the phononic system in $\mathrm{NbN}$, $C_{\mathrm{ph}}$. While the former quantity is straightforward related to the density of states at the Fermi level in $\mathrm{NbN}$ and can be obtained by all transport means as explained below, the latter is not so easily accessible. Here we infer the lower bound for $C_{\mathrm{ph}}$, and hence the upper bound for $C_{e}^{n} / C_{\mathrm{ph}}$, from the measured $Z$ using Eq. (3) as follows:

$$
\frac{C_{e}^{n}}{C_{\mathrm{ph}}}<C_{e}^{n} \frac{Z}{\tau_{\mathrm{esc}}}<C_{e}^{n} \frac{Z}{\tau_{\mathrm{esc}}^{\text {low }}}
$$

where $\tau_{\text {esc }}^{\text {low }}$ is the lower bound for the acoustic phonon escape time in dirty $\mathrm{NbN}$ films adopted from the literature. Remarkably, we find that at $T=T_{c}$ such an upper bound is close to the recent theoretical lower bound [13] expected for the same quantity in our SSPD. This observation is one of the main results of our paper, along with the absolute measurement of $Z$.

\section{B. Sample}

We perform our experiments on a $5.5 \pm 0.5 \mathrm{~nm}$ thick, 100 -nm wide $\mathrm{NbN}$ nanowire patterned in a meander shape. The meander covers an area of $15 \times 15 \mu \mathrm{m}^{2}$, which roughly corresponds to the size of a light spot at the end of the optical fiber. On top of the sample a 450-nm-thick antireflection $\mathrm{Al}_{2} \mathrm{O}_{3} / \mathrm{Si} / \mathrm{Al}_{2} \mathrm{O}_{3}$ coating is deposited. To increase absorption by the sample, we place an optical cavity between the $\mathrm{NbN}$ meander and the $\mathrm{SiO}_{2} / \mathrm{Si}$ substrate, which consists of a 70-nm-thick bottom Au mirror and a $200 \mathrm{~nm}$-thick dielectric $\mathrm{Si}_{3} \mathrm{~N}_{4}$ layer [3]. The device has $T_{c}$ of $7.84 \mathrm{~K}$. The sheet resistance $R_{\square}$ is measured at room temperature by using a four-point probe technique for the film before lithographic processing. The resistivity $\rho$ is derived from $\rho=R_{\square} d$, where the thickness $d$ is determined from the deposition time with use of a calibrated deposition rate.

The resistivity in the normal state, $\rho_{n}$, relevant for the diffusivity and elastic scattering in the superconducting state is determined from the resistivity above the resistive transition at $T=20 \mathrm{~K}$. The measured resistance ratio, $\rho_{(300 \mathrm{~K})} / \rho_{n}=R_{(300 \mathrm{~K})} / R_{(20 \mathrm{~K})}=0.8$, is used to convert the room-temperature measurement to an actual number, which is $4.4 \times 10^{-6} \Omega \mathrm{m}$. In contrast to conventional 
TABLE I. Parameters of the NbN film, with $d$ the thickness, $V$ the volume of the device, $T_{c}$ determined from the midpoint of the resistive transition, $I_{c}$ the superconducting critical current at $T=1.7 \mathrm{~K}, R_{\square}$ the sheet resistance at room temperature, and $\rho_{n}$ the resistivity at $T=20 \mathrm{~K} . D$ is the diffusivity extracted from the temperature dependence of resistance under a magnetic field and $N(0)$ is the single-spin density of states obtained from the transport data (see Sec. II E).

\begin{tabular}{lccccccc}
\hline \hline $\begin{array}{l}d \\
(\mathrm{~nm})\end{array}$ & $\begin{array}{c}V \\
\left(\mathrm{~cm}^{3}\right)\end{array}$ & $\begin{array}{c}T_{c} \\
(\mathrm{~K})\end{array}$ & $\begin{array}{c}I_{c} \\
(\mu \mathrm{A})\end{array}$ & $\begin{array}{c}R_{\square} \\
(\Omega / \square)\end{array}$ & $\begin{array}{c}\rho_{n} \\
(\Omega \mathrm{m})\end{array}$ & $\begin{array}{c}D \\
\left(\mathrm{~cm}^{2} / \mathrm{s}\right)\end{array}$ & $\begin{array}{c}N(0) \\
\left(\mathrm{eV}^{-1} \mathrm{~nm}^{-3}\right)\end{array}$ \\
\hline 5.5 & $6.6 \times 10^{-13}$ & 7.84 & 13 & 640 & $4.4 \times 10^{-6}$ & 0.35 & 20.3 \\
\hline \hline
\end{tabular}

metals, the resistivity at cryogenic temperatures is higher than at room temperature. The parameters of the device are summarized in Table I.

\section{Absorbed power}

Knowledge of the absolute absorbed power $P_{\mathrm{abs}}$ is a key ingredient to determine the thermal resistance $Z$. In general, only a fraction of the input optical power $P_{\text {in }}$ is absorbed by the detector. In our experiment, $\eta_{\text {couple }}=P_{\text {abs }} / P_{\text {in }}<1$ is mainly caused by imperfections of the multilayer antireflection coating and misalignment of the light spot at the end of the optical fiber with respect to the detector area, which is quantified as described below.

Even in an idealized SSPD free from all sorts of coupling losses $\left(\eta_{\text {couple }}=1\right)$ the absorption of a single-photon does not necessary result in a detection event, which is a temporary switching of the device into the resistive state. In dirty superconductors with short thermalization time, including $\mathrm{NbN}$ in the present experiment, the underlying switching mechanism is most adequately described with a HS model [13]. In this case, shortly after the photon absorption, the superconducting gap inside the HS is suppressed, giving rise to the nucleation of a vortex-antivortex pair inside the superconducting strip or the entrance of a single vortex from its nearest edge [12]. Whether this promotes the detection event or not depends on the position, shape, and volume of the HS. In addition, the internal efficiency, which determines the single-photon detection probability of the idealized SSPD, should increase with the bias current $I$ owing to a current-crowding effect and heating of the superconductor by moving unbound vortices $[12,27]$. Therefore, a saturation of the photon count rate as a function of $I$, observed in the best SSPDs [1-3,28,29], indicates that each absorbed photon is detected. This is the regime of unit internal efficiency, as verified both theoretically $[13,27,30]$ and in experiments with minimized coupling losses [1-3].

Tuning our device in the regime of unit internal efficiency, we are able to quantify the coupling efficiency $\eta_{\text {couple }}$. This experiment is designed as follows [see the inset in Fig. 1(b) for a schematic of our setup]. The SSPD is cooled to $T_{b}=1.7 \mathrm{~K}$, where it shows its best performance, and is illuminated with $1.2-\mathrm{pW} \mathrm{cw}$ optical radiation of wavelength $1.55 \mu \mathrm{m}$ through a single-mode fiber attached on the top of the detector. At a finite dc bias current, the absorption of a single photon results in a voltage pulse, further amplified by broadband microwave roomtemperature amplifiers (50 dB gain) and counted with an Aligent 53131A pulse counter. The input optical power is measured at the entrance of the dipstick with an Ophir PD300-IRG calibrated power meter. The incident optical power $P_{\text {in }}$ is attenuated by an EXFO FVA-600 optical variable attenuator. The coupling efficiency of the SSPD varies by approximately $20 \%$ depending on the polarization of the incident light, and the experiment is performed at the maximum response. The choice of the polarization is irrelevant for the measured $Z$. Figure 1(b) shows the biascurrent dependence of the measured detection efficiency, $D_{E}$, which is the ratio of the counted and input photon numbers. The observed saturation of $D_{E}$ at increasing current [see the dashed line in Fig. 1(b)] is evidence of the regime of unit internal efficiency. Here the photon detection is limited only by the coupling efficiency, and we obtain $\eta_{\text {couple }}=D_{E} \approx 52 \%$, which is used to calculate $P_{\text {abs }}$ and $Z$ below.

\section{Thermal resistance}

The final step to obtain the thermal resistance, $Z=$ $\delta T_{e} / P_{\mathrm{abs}}$, is the measurement of the variation of the electron temperature in phase with the modulated optical power. We track the small oscillation $\delta T_{e}$ by measuring the oscillating voltage on the SSPD biased with the direct current $I$ using the standard relation $\delta U=I\left(\partial R / \partial T_{e}\right) \delta T_{e}$. We expect that $T_{e}$ is the only parameter that controls the detector resistance in our experiment; therefore, $\partial R / \partial T_{e}$ is the same as the derivative $d R / d T$ determined from the temperature dependence of the resistance in equilibrium, when the bath and electron temperatures coincide, $T=T_{e}=T_{b}$. Obviously, our approach is sensitive to $\delta T_{e}$ only in the vicinity of the resistive transition, where the temperature dependence is strongest, and hence the resolution is limited by the width of the transition. To expand the temperature range available, we use a perpendicular magnetic field that shifts the transition to lower temperatures and simultaneously smears it out.

Figures 2(a) and 2(b) show, respectively, the temperature dependencies of the sample resistance in equilibrium and of the measured voltage modulation under the modulated optical radiation for a set of magnetic fields up to $B=4 \mathrm{~T}$. The data in Fig. 2(a) are smoothed and the 


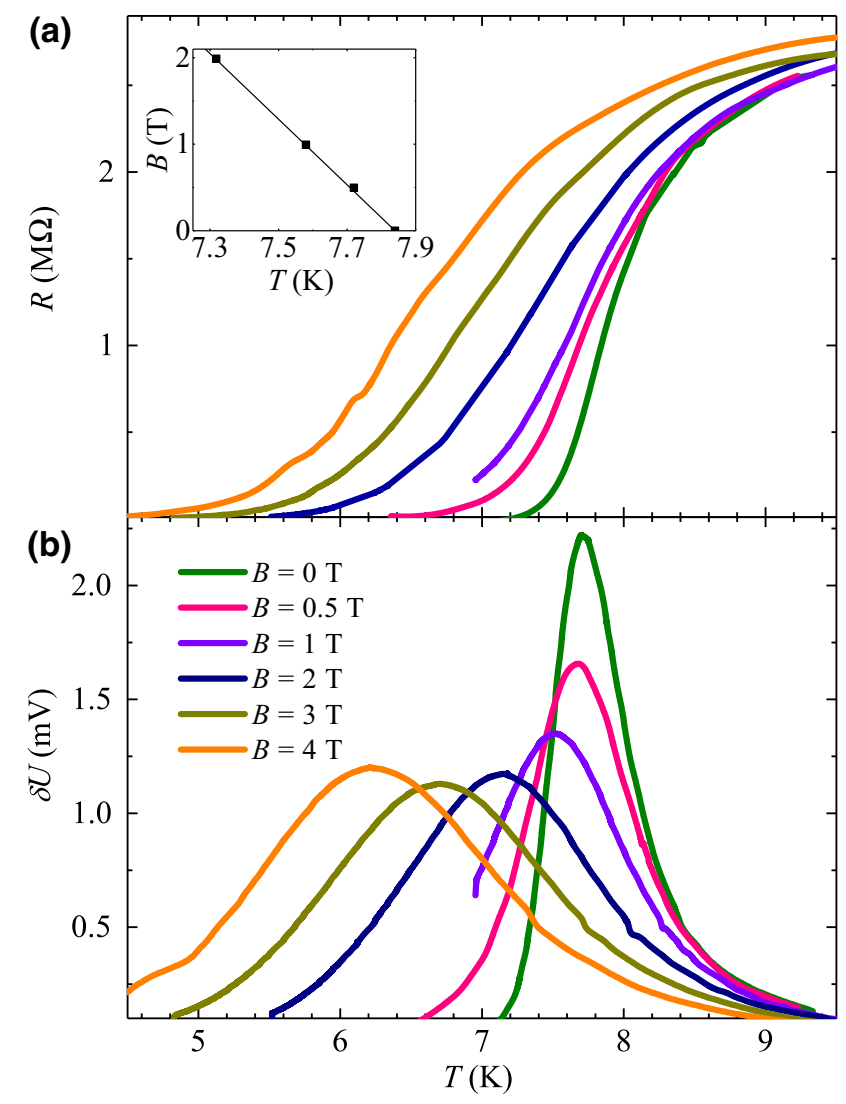

FIG. 2. The temperature dependencies of the sample resistance in equilibrium (a) and of the measured voltage response under optical radiation (b) for a set of magnetic fields (see the legend). For (b) a laser wavelength of $1.55 \mu \mathrm{m}$, an input laser power of $P_{\text {in }}=320 \mathrm{nW}$, and a dc bias current of $I=100 \mathrm{nA}$ are used. The inset shows the temperature dependence of the upper critical field $B_{c 2}(T)$ used to evaluate the density of states in $\mathrm{NbN}$. The temperature dependencies in (a),(b) are obtained by our measuring the signals during warm-up at discrete temperatures (15-20 points per curve). At each point the temperature was stabilized for at least $10 \mathrm{~min}$ with an accuracy of about $\pm 1 \mathrm{mK}$.

derivative $d R / d T$ is obtained numerically. With increasing $B, T_{c}$ decreases and the resistive transition smears out. The measured $\delta U$ changes accordingly. While the input optical power remains constant throughout the experiment, $\delta U$ follows a bell-shaped curve, which is slightly different from the calculated $d R / d T$ (not shown). As demonstrated below, for $B \geq 1 \mathrm{~T}$ this difference is fully captured by the temperature dependence of the thermal resistance $Z(T)$.

The measured $\delta U$ in Fig. 2(b) translates to a temperature variation as small as $\delta T_{e} \sim 10 \mathrm{mK}$. This value, averaged both in time and throughout the device, is much smaller than the characteristic temperature variation $\delta T_{e}^{\mathrm{HS}}$ within the HS formed after photon absorption. Obviously, the validity of our approach is based on the assumption that the temperature dependencies of both $Z(T)$ and $R(T)$ can be approximated as linear on the scale of $\delta T_{e}^{\mathrm{HS}} \cdot \delta T_{e}^{\mathrm{HS}}=$ $\alpha E_{\mathrm{ph}} /\left(C_{e}^{n} V\right)$, where $\alpha$ is the assumed fraction of the photon energy $E_{\mathrm{ph}}=0.8 \mathrm{eV}$ that goes into the electron system, $C_{e}^{n}$ is the normal-state thermal capacity of the electron system, and $V$ is the HS volume. The HS volume is $V=2 r_{\mathrm{HS}} w d$, where $d=5.5 \mathrm{~nm}$ and $w=100 \mathrm{~nm}$ are, respectively, the thickness and the width of the superconducting film and $r_{\mathrm{HS}}=2(D \tau)^{1 / 2} \approx 160 \mathrm{~nm}>w$ is the HS radius, obtained from the experimental value of the diffusion coefficient $D$ and relaxation time $\tau \sim 50$ ps typical for $\mathrm{NbN}$. On the basis of the data in Sec. II E and with $\alpha=0.4$, which correlates with our analysis below, we estimate $\delta T_{e}^{\mathrm{HS}} \approx 1.5$ $\mathrm{K}$. This value corresponds to much stronger heating than the measured time- and device-averaged value of $\delta T_{e} \sim 10$ $\mathrm{mK}$ and is comparable to the width of the resistive transition in a magnetic field. Still, the fact that the data for different $B$ coincide within the experimental uncertainty (Fig. 3) indicates that our assumption of the linear response is reasonable. Hence, the inevitable spatial and temporal inhomogeneity of the heating and the associated resistive response of the electron system are irrelevant for the purpose of $Z(T)$ measurement.

\section{E. Density of states and electron heat capacity}

Transport measurements also contain information on the temperature dependence of the upper critical field $B_{c 2}(T)$ in our sample, which is determined from the maxima of $d R / d T$ and plotted in the inset in Fig. 2(a). As usual, from the linear part of this temperature dependence we extract the diffusion constant in $\mathrm{NbN}, D=$ $-\left(4 k_{B} / \pi e\right)\left(d B_{c 2} / d T\right)^{-1}=0.35 \mathrm{~cm}^{2} / \mathrm{s}$. Next, using the Sommerfeld model for free-electron gas [31], we obtain a single-spin density of states at the Fermi level, $N(0)=$ $\left(2 \rho_{n} D e^{2}\right)^{-1} \approx 20.3 \mathrm{eV}^{-1} \mathrm{~nm}^{-3}$, where $\rho_{n}$ is the normalstate resistance at $T=20 \mathrm{~K}$, and evaluate the normal-state thermal capacitance, $C_{e}^{n}=2 \pi k_{B}^{2} N(0) T / 3$, where $k_{B}$ is the Boltzmann constant. We believe that this ab initio estimate is well justified in our dirty $\mathrm{NbN}$ system since the electron mean free path is about $1 \AA$, and thereby the crystalline order is destroyed and a possible Fermi surface anisotropy is negligible.

\section{RESULTS}

In Fig. 3 we present the temperature dependence of the measured thermal resistance $Z$ of our NbN SSPD for a set of magnetic field $B$ values obtained as described in Sec. IID. At each setting of $B$ (see the legend) the data points cover a temperature range corresponding to the width of a respective $\delta U$ peak in Fig. 2(b). For different $B \geq 1 \mathrm{~T}$, the data ranges sufficiently overlap and demonstrate good consistency, indicating that our two-temperature model adequately describes the power response of the SSPD in finite magnetic fields. Altogether, these data cover the range between 5 and $9 \mathrm{~K}$ and enable us to extract a power-law temperature dependence of the form $Z \propto T^{-3}$ (see the dashed guide line in Fig. 3). 


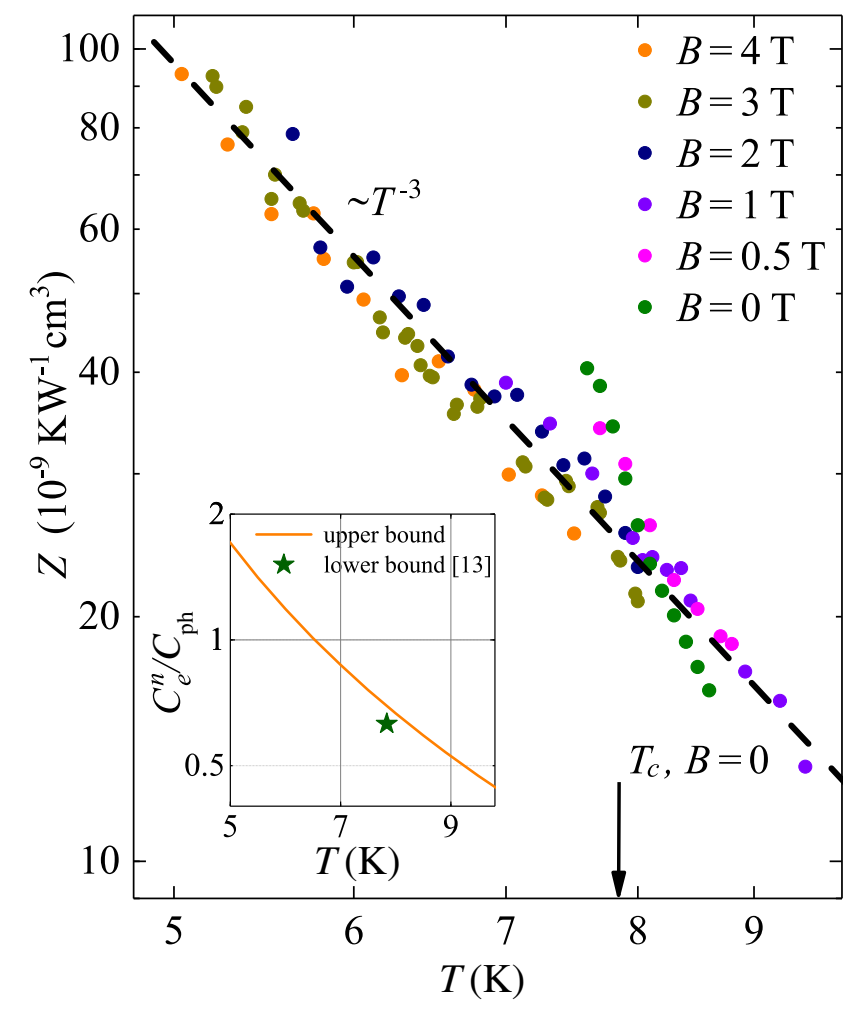

FIG. 3. Temperature dependence of the thermal resistance $Z$ for a $\mathrm{NbN}$ SSPD at the resistive transition. The experimental datasets are obtained from the measured power response of our SSPD in different magnetic fields, as explained in Sec. II D. The dashed guide line represents the power-law dependence $Z \propto T^{-3}$ observed. The $B=0$ critical temperature of the resistive transition is marked by an arrow. The inset shows the ratio of the electron (normal state) and phonon thermal capacitances as a function of temperature. The solid line is the upper bound imposed by our experiment, as discussed in the text. The star is the lower bound, which follows from the theory in Ref. [13] for parameter values similar to those of our SSPD.

Notable in Fig. 3 are sizeable deviations for the two lowest magnetic fields, for which we find that the temperature dependence becomes considerably stronger as $B$ tends to zero. In this respect, our observation is reminiscent of the $B=0$ peculiarities of the relaxation time observed long ago in dirty $\mathrm{Nb}$ films [32] and more recently in borondoped diamond films [15] by the AMAR technique. The origin of this puzzling behavior was associated [15] with the longitudinal relaxation time in nonequilibrium superconductivity [33]. This physics is certainly beyond the applicability of our two-temperature model; therefore, the corresponding two datasets in Fig. 3 are discarded in our analysis of the temperature dependence of $Z$.

As seen from Eq. (3), in principle, knowledge of $Z(T)$ allows us to extract simultaneously both the electronphonon and the phonon-escape thermal resistances, provided their temperature dependencies are sufficiently different. Unfortunately, this is difficult to realize here.
According to the literature, for thin $\mathrm{NbN}$ films $\tau_{e \text {-ph }} \propto$ $T^{-1.6}[17,18]$ and $\tau_{\text {esc }} \propto d[34-36]$, with numerical prefactors dependent on the film quality, substrate, and perhaps other experimental details. Hence, assuming for the thermal capacities $C_{e} \propto T$ and $C_{\mathrm{ph}} \propto T^{3}$, we expect the functional dependencies of $Z_{e \text {-ph }} \propto T^{-2.6}$ and $Z_{\text {esc }} \propto T^{-3}$, which are very close to each other and to the experimental $Z(T)$ in Fig. 3.

The uncertainty of the available data on the timescales $\tau_{e \text {-ph }}$ and $\tau_{\text {esc }}[17,18,34-36]$, as well as the lack of independent measurement of $C_{e}$ at the resistive transition, makes it unreliable to discriminate between the two contributions to $Z$ in Eq. (3). Instead, using the evaluated normal-state electron thermal capacity $\left(C_{e}^{n}\right.$; see Sec. II E) and the experimental $Z(T)$, we determine the upper boundary for the ratio $C_{e}^{n} / C_{\mathrm{ph}}$. This is achieved via relation (4), in which the lower bound of the acoustic phonon escape time in dirty $\mathrm{NbN}$ films is adopted from Ref. [26], $\tau_{\mathrm{esc}}^{\text {low }} / d=8 \mathrm{ps} / \mathrm{nm}$. The linear dependencies of the relaxation time on $d$, established in Refs. [35,36], along with the typical timescale $\tau_{\text {esc }} \gg d / s$, where $s$ is the sound velocity, imply extremely short mean free paths for acoustic phonons in thin $\mathrm{NbN}$ films. Thereby the scenario of full internal reflection of phonons proposed recently in WSi [21] is not applicable here. The result of our analysis is shown in the inset in Fig. 3 by the solid line with the temperature dependence of $C_{e}^{n} / C_{\mathrm{ph}} \propto T^{-2}$ and the absolute value $C_{e}^{n} / C_{\mathrm{ph}} \sim 1$, which is in reasonable agreement with the estimates based on the Debye model in $\mathrm{NbN}$, as discussed below. Most intriguing is a comparison with the recent SSPD theory [13], which relates $C_{e}^{n} / C_{\mathrm{ph}}$ to the detection efficiency. We observe that in the vicinity of the $B=0$ resistive transition, the experimental upper boundary is close to the theoretical lower boundary for SSPDs with unit internal efficiency (see the star in the inset in Fig. 3). This finding is also discussed below.

\section{DISCUSSION}

The measurement of the absolute value of $Z(T)$ in Fig. 3 is the central experimental result of our work. The data are consistent with the functional dependence as well as the order-of-magnitude estimate based on the available data on the relaxation timescales $\tau_{e \text {-ph }}$ and $\tau_{\text {esc }}$ in $\mathrm{NbN}$ [17,18,34-36] and the estimates of $C_{e}$ and $C_{\mathrm{ph}}$. This justifies our experimental approach, which has two clear advantages. First, the use of the optical frequencies ensures uniform absorption efficiency by a potentially inhomogeneous superconducting film, in contrast, for example, to a much-simpler Joule-heating approach [37]. Second, the device- and setup-dependent radiation-coupling efficiency can be accurately calibrated in the regime of unit internal efficiency of our SSPD, providing knowledge of the absolute absorbed optical power. The main drawback of our approach is the temperature resolution limited by the 
width of the resistive transition, which can be overcome by shifting of the transition with the help of a magnetic field, yet with certain reservations. In particular, it is not clear at present whether the deviations of $Z(T)$ around $B=0$ from the general trend $Z \propto T^{-3}$ in Fig. 3 capture the real changes in $Z$ or manifest a failure of the two-temperature model caused, for example, by nonequilibrium phenomena [15] or by the vicinity of the BKT transition.

The lower bound imposed on the phonon thermal capacity, $C_{\mathrm{ph}}>\tau_{\mathrm{esc}}^{\text {low }} / Z$, and translated into the upper bound of $C_{e}^{n} / C_{\mathrm{ph}}$ in Fig. 3 (inset, solid line) is also an intriguing result. With the Debye model $C_{\mathrm{ph}}=$ $\left(12 \pi^{4} / 5\right) N_{i} k_{B}\left(T / \Theta_{D}\right)^{3}$ [38], where $N_{i}$ is the ion density, we obtain an estimate of the Debye temperature $\left(\Theta_{D}<\right.$ $430 \mathrm{~K})$ that compares reasonably with some reported values $\left(\Theta_{D}=174-363 \mathrm{~K}[39-42]\right)$.

Luckily, more conclusive is a comparison with the recent photon-generated-superconducting-vortex model from Ref. [13]. In our SSPD operating in the unit-internalefficiency regime at $1.7 \mathrm{~K}$, the ratio of the measured superconducting critical current and the theoretical depairing current, $I_{\mathrm{dep}}$, does not exceed 0.6. Hence, the saturation of detection efficiency in Fig. $1 \mathrm{~b}$ occurs at $I \approx$ $0.4 I_{\text {dep. }}$. For $\mathrm{NbN}$ SSPD devices of similar width $w=$ $128 \mathrm{~nm}, T / T_{c} \approx 0.2$, and $E_{\mathrm{ph}}=0.8 \mathrm{eV}$, the photongenerated-superconducting-vortex model predicts saturation at $I / I_{\mathrm{dep}} \approx 0.5$ provided $\left.\left(C_{e}^{n} / C_{\mathrm{ph}}\right)\right|_{T=T_{c}} \geq 0.63$ (see the upper dataset in Fig. 12 in Ref. [13] extrapolated to lower $T / T_{c}$ ). This lower bound, marked by the star in the inset in Fig. 3, is just about $15 \%$ below the solid line, implying that the actual ratio $C_{e}^{n} / C_{\mathrm{ph}}$ is quite close to the experimental upper bound. Thus, we speculate that, first, our SSPD resides on the boundary of the best materials for single-photon detection and, second, the thermal resistance between $\mathrm{NbN}$ and the substrate is dominated by the phonon escape term; that is, $Z \approx Z_{\text {esc }} \gg Z_{e \text {-ph }}$ in Eq. (3). The former conjecture correlates with an empiric finding that $\mathrm{NbN}$-based SSPDs with unit internal efficiency are trickier to fabricate than their analogs based on WSi, which have a much larger thermal-capacity ratio [13]. The latter is less obvious and implies that $\tau_{\mathrm{esc}} \gg \tau_{\mathrm{ph}-e}$; that is, the phonon escape time into the substrate is much longer than its lifetime limited by the electron-phonon interaction. Although it is difficult to draw a definitive conclusion from the present experiment, such a strong electron-phonon coupling regime might serve to explain the well-known fact [21,26,34-36] that $\tau_{\text {esc }}$ in dirty superconducting films is more than one order of magnitude longer than the time of flight of a ballistic phonon in the direction of a substrate.

In summary, we investigate the thermal properties of a $\mathrm{NbN}$ single-photon detector capable of unit internal detection efficiency in the infrared range. The key of our approach is the independent calibration of the apparatus's coupling losses, which provides knowledge of the optical power absorbed by the $\mathrm{NbN}$ film. Using a resistive response of the device for thermometry in the vicinity of the resistive transition in zero and finite magnetic field, we extract the thermal resistance $Z$ and analyze its temperature dependence with a two-temperature model. Our results provide an estimate of the ratio of electron and phonon heat capacities and suggest that the thermal resistance is dominated by the process of phonon escape from the $\mathrm{NbN}$ film into the substrate.

\section{ACKNOWLEDGMENTS}

We acknowledge discussions with D.Yu. Vodolazov and E.S. Tikhonov. This research was supported by the Russian Science Foundation (Project No. 17-72-30036).

[1] F. Marsili, V. B. Verma, J. A. Stern, S. Harrington, A. E. Lita, T. Gerrits, I. Vayshenker, B. Baek, M. D. Shaw, R. P. Mirin, and S. W. Nam, Detecting single infrared photons with 93\% system efficiency, Nat. Photonics 7, 210 (2013).

[2] O. Kahl, S. Ferrari, V. Kovalyuk, G. N. Goltsman, A. Korneev, and W. H. P. Pernice, Waveguide integrated super conducting single-photon detectors with high internal quantum efficiency at telecom wavelengths, Sci. Rep. 5, 10941 (2015).

[3] K. Smirnov, A. Divochiy, Yu. Vakhtomin, P. Morozov, Ph. Zolotov, A. Antipov, and V. Seleznev, NbN single-photon detectors with saturated dependence of quantum efficiency, Supercond. Sci. Technol. 31, 035011 (2018).

[4] A. Pearlman, A. Cross, W. Slysz, J. Zhang, A. Verevkin, M. Currie, A. Korneev, P. Kouminov, K. Smirnov, B. Voronov, G. Gol'tsman, and R. Sobolewski, Gigahertz counting rates of $\mathrm{NbN}$ single-photon detectors for quantum communications, IEEE Trans. Appil. Supercond. 15, 579 (2005).

[5] C. M. Natarajan, M. G. Tanner, and R. H. Hadfield, Superconducting nanowire single-photon detectors: Physics and applications, Supercond. Sci. Technol. 25, 063001 (2012).

[6] H. Bartolf, Fluctuation Mechanisms in Superconductors (Springer Fachmedien, Wiesbaden, 2016).

[7] T. M. Klapwijk and A. V. Semenov, Engineering Physics of superconducting hot-electron bolometer mixers, IEEE Trans. Terahertz Sci. Technol. 7, 627 (2017).

[8] V. F. Gantmakher and V. T. Dolgopolov, Superconductorinsulator quantum phase transition, Uspekhi Fizicheskih Nauk 53, 1 (2010).

[9] M. R. Beasley, J. E. Mooij, and T. P. Orlando, Possibility of Vortex-Antivortex Pair Dissociation in Two-Dimensional Superconductors, Phys. Rev. Lett. 42, 1165 (1979).

[10] B. I. Halperin and David R. Nelson, Resistive transition in superconducting films, J. Low Temp. Phys. 36, 599 (1979).

[11] G. N. Goltsman, O. Okunev, G. Chulkova, A. Lipatov, A. Semenov, K. Smirnov, B. Voronov, A. Dzardanov, C. Williams, and R. Sobolewski, Picosecond superconducting single-photon optical detector, Appl. Phys. Lett. 79, 705 (2001).

[12] A. N. Zotova and D. Yu. Vodolazov, Intrinsic detection efficiency of superconducting nanowire single photon detector 
in the modified hot spot model, Supercond. Sci. Technol. 27, 125001 (2014).

[13] D. Yu. Vodolazov, Single-Photon Detection by a Dirty Current-Carrying Superconducting Strip based on the Kinetic-Equation Approach, Phys. Rev. Appl. 7, 034014 (2017).

[14] Yu. P. Korneeva, D. Yu. Vodolazov, A. V. Semenov, I. N. Florya, N. Simonov, E. Baeva, A. A. Korneev, G. N. Goltsman, and T. M. Klapwijk, Optical Single-Photon Detection in Micrometer-Scale NbN Bridges, Phys. Rev. Appl. 9, 064037 (2018).

[15] A. Kardakova, A. Shishkin, A. Semenov, G. N. Goltsman, S. Ryabchun, T. M. Klapwijk, J. Bousquet, D. Eon, B. Sacépé, Th. Klein, and E. Bustarret, Relaxation of the resistive superconducting state in boron-doped diamond films, Phys. Rev. B 93, 064506 (2016).

[16] E. M. Gershenzon, M. E. Gershenzon, G. N. Goltsman, A. M. Lyulkin, A. D. Semenov, and A. V. Sergeev, Sov. Phys. JETP 70, 505 (1990).

[17] Yu. P. Gousev, G. N. Goltsman, A. D. Semenov, E. M. Gershenzon, R. S. Nebosis, M. A. Heusinger, and K. F. Renk, Broadband ultrafast superconducting NbN detector for electromagnetic radiation, J. Appl. Phys. 75, 3695 (1994).

[18] Yu. P. Gousev, A. D. Semenov, G. N. Goltsman, A. V. Sergeev, and E. M. Gershenzon, Electron-phonon interaction in disordered NbN films, Phys. B: Condens. Matter 194-196, 1355 (1994).

[19] A. Kardakova, M. Finkel, D. Morozov, V. Kovalyuk, P. An, C. Dunscombe, M. Tarkhov, P. Mauskopf, T. M. Klapwijk, and G. Goltsman, The electron-phonon relaxation time in thin superconducting titanium nitride films, Appl. Phys. Lett. 103, 252602 (2013).

[20] K. S. Ilin, N. G. Ptitsina, A. V. Sergeev, G. N. Gol'tsman, E. M. Gershenzon, B. S. Karasik, E. V. Pechen, and S. I. Krasnosvobodtsev, Interrelation of resistivity and inelastic electron-phonon scattering rate in impure NbC films, Phys. Rev. B 57, 15623 (1998).

[21] M. V. Sidorova, A. G. Kozorezov, A. V. Semenov, Yu. P. Korneeva, M. Yu. Mikhailov, A. Yu. Devizenko, A. A. Korneev, G. M. Chulkova, and G. N. Goltsman, Nonbolometric bottleneck in electron-phonon relaxation in ultrathin WSi films, Phys. Rev. B 97, 184512 (2018).

[22] V. N. Ryzhov, E. E. Tareyeva, Yu. D. Fomin, and E. N. Tsiok, Berezinskii-Kosterlitz-Thouless transition and two-dimensional melting, Uspekhi Fizicheskih Nauk 187, 921 (2017).

[23] N. Perrin and C. Vanneste, Dynamic behaviour of a superconductor under time-dependent external excitation, J. Phys. 48, 1311 (1987).

[24] N. Perrin, Phonons in thin optically irradiated superconductors; spectrum and possible definition of a phonon temperature, J. Phys. 41, 615 (1980).

[25] A. Semenov, B. Günther, U. Böttger, H.-W. Hübers, H. Bartolf, A. Engel, A. Schilling, K. Ilin, M. Siegel, R. Schneider, D. Gerthsen, and N. A. Gippius, Optical and transport properties of ultrathin $\mathrm{NbN}$ films and nanostructures, Phys. Rev. B 80, 054510 (2009).

[26] A. D. Semenov, R. S. Nebosis, Yu. P. Gousev, M. A. Heusinger, and K. F. Renk, Analysis of thenonequilibrium photoresponse of superconducting films to pulsed radiation by use of a two-temperature model, Phys. Rev. B 52, 581 (1995).

[27] L. N. Bulaevskii, M. J. Graf, and V. G Kogan, Vortexassisted photon counts and their magnetic field dependence in single-photon superconducting detectors, Phys. Rev. B 85, 014505 (2012).

[28] B. Baek, A. E. Lita, V. Verma, and S. W. Nam, Superconducting a $-\mathrm{W}_{x} \mathrm{Si}_{1-x}$ nanowire single-photon detector with saturated internal quantum efficiency from visible to 1850 nm, Appl. Phys. Lett. 98, 251105 (2011).

[29] F. Marsili, F. Najafi, E. Dauler, F. Bellei, X. Hu, M. Csete, R. J. Molnar, and K. K. Berggren, Single-photon detectors based on ultranarrow superconducting nanowires, Nano Lett. 11, 2048 (2011).

[30] S. Ferrari, O. Kahl, V. Kovalyuk, G. N. Goltsman, A. Korneev, and W. H. P. Pernice, Waveguide-integrated single- and multi-photon detection at telecom wavelengths using superconducting nanowires, Appl. Phys. Lett. 106, 151101 (2015).

[31] C. Kittel, Introduction to Solid State Physics (Wiley India Pvt. Limited, New Delhi, 2007).

[32] E. M. Gershenzon, M. E. Gershenzon, G. N. Goltsman, A. D. Semenov, and A. V. Sergeev, Heating of electrons in a superconductor in the resistive state by electromagnetic radiation, Sov. Phys. JETP 59, 442 (1984).

[33] M. Tinkham, Introduction to Superconductivity. Dover Books on Physics Series (Dover Publications, New York, 2004).

[34] D. Rall, P. Probst, M. Hofherr, S. Wünsch, K. Ilin, U. Lemmer, and M. Siegel, Energy relaxation time in $\mathrm{NbN}$ and YBCO thin film sunder optical irradiation, J. Phys.: Conf. Ser. 234, 042029 (2010).

[35] D. Henrich, Influence of Material and Geometry on the Performance of Superconducting Nanowire SinglePhoton Detectors (KIT Scientific Publishing, Karlsruhe, 2013).

[36] S. Cherednichenko, P. Yagoubov, K. Ilin, G. Goltsman, and E. Gershenzon, in 1997 27th European Microwave Conference (IEEE, Cambridge, 1997).

[37] A. Brown, M. W. Zemansky, and H. A. Boorse, The superconducting and normal heat capacities of niobium, Phys. Rev. 92, 52 (1953).

[38] N. W. Ashcroft and N. D. Mermin, Solid State Physics (Harcourt School, New York, 1987).

[39] S. P. Chockalingam, M. Chand, J. Jesudasan, V. Tripathi, and P. Raychaudhuri, Superconducting properties and Hall effect of epitaxial NbN thin films, Phys. Rev. B 77, 214503 (2008).

[40] T. H. Geballe, B. T. Matthias, J. P. Remeika, A. M. Clogston, V. B. Compton, J. P. Maita, and H. J. Williams, High temperature SP-band superconductors, Phys. Phys. 2, 293 (1966).

[41] P. Roedhammer, E. Gmelin, W. Weber, and J. P. Remeika, Observation of phonon anomalies in $\mathrm{NbC}_{x} \mathrm{~N}_{1-x}$ alloys, Phys. Rev. B 15, 711 (1977).

[42] C. Geibel, H. Rietschel, A. Junod, M. Pelizzone, and J. Muller, Electronic properties, phonon densities of states and superconductivity in $\mathrm{Nb}_{1-x} \mathrm{~V}_{x} \mathrm{~N}$, J. Phys. F: Metal Phys. 15, 405 (1985). 